\title{
Aggressive Giant Fibroepithelial Lesion with Unusual Vascular Stroma-A Case Report
}

Wedad Hanna, F.R.C.P.C., J. AL-Maghrabi, M.D., Abha Malik, M.D.

Department of Anatomical Pathology, Sunnybrook and Women's College Health Science Center, University of Toronto, Toronto, Canada

The stroma of fibroadenoma and phyllodes tumor usually consists of fibroblastic proliferation. Rarely the stroma contains bundles of smooth muscle. Pseudoangiomatous hyperplasia of the mammary stroma has been described in fibroadenomas. However, true benign vascular stroma has not been reported. We report a case of a 34-year-old Chinese woman who presented with a large mass occupying the entire left breast. Left mastectomy was performed and showed a large, well-circumscribed, lobulated, rubbery-firm tumor measuring $13 \times 10$ $\times 6 \mathrm{~cm}$. Microscopic examination revealed a fibroepithelial tumor formed by an organoid pattern of ductal structures with a very striking stromal appearance composed of extensive vascular proliferation and that demonstrated strong immunoreactivity for CD31, CD34, and Factor VIII. Ultrastructural examination revealed intercellular junctions, basal lamina, pinocytotic vesicles, and Weibel-Palade bodies in the cells lining the vascular spaces, confirming their endothelial nature. These findings rule out the diagnosis of pseudoangiomatous hyperplasia. The patient developed local recurrence a year later, and the resection showed malignant phyllodes tumor with ductal carcinoma in situ. The extensive vascular stroma noted in the primary tumor may have played a role in the malignant transformation of the epithelial and stromal components in this tumor.

KEY WORDS: Angiogenesis, Giant fibroepithelial lesion with vascular stroma, Phyllodes tumor, Vascular breast lesions.

Mod Pathol 2003;16(8):823-827

Copyright ( $\odot 2003$ by The United States and Canadian Academy of Pathology, Inc.

VOL. 16, NO. 8, P. 823, 2003 Printed in the U.S.A.

Date of acceptance: February 27, 2003.

Address reprint requests to: Wedad Hanna, F.R.C.P.C., Anatomic Pathology, Sunnybrook and Women's College, Health Sciences Center, 2075 Bayview Avenue, M5S 1B2, Toronto, Ontario, Canada 30322; fax: 416-4804271; e-mail: wedad.hanna@swchsc@on.ca.

DOI: 10.1097/01.MP.0000075644.61258.85
A 34-year-old Chinese woman presented with a large mass occupying the entire left breast. Left mastectomy was performed that showed a remarkable fibroepithelial lesion with stroma showing extensive vascular proliferation. The patient developed a local recurrence a year later, and the resection was diagnosed as malignant phyllodes tumor with ductal carcinoma in situ. The patient had had right mastectomy 5 years ago in China before coming to Canada. No information on the pathology could be obtained.

\section{MATERIALS AND METHODS}

All specimens were received fixed in $10 \%$ neutral formalin. Paraffin-embedded blocks and sections were prepared in the conventional manner and stained with hematoxylin and eosin. Immunohistochemistry was performed using avidin-biotin complex technique $(\mathrm{ABC})$ with appropriate positive and negative controls. The immunohistochemistry panel included antibodies against CD31 (DAKO, 1/50), CD34, Factor VIII (DAKO, 1/1200), S100 (DAKO, 1/500), actin (Enzo, 1/50), desmin (Biogenex, 1/50), vimentin (Novocastra, 1/75), E-cadherin (Transduction, 1/2500), and CAM 5.2 (Becton Dickinson, 1/8).

For electron microscopy, paraffin-embedded, formalin-fixed tissue was postfixed in osmium, and ultrathin sections were cut after appropriate area was selected from semithin sections.

\section{Pathologic Findings}

The initial breast tumor was large, well-defined, lobulated, rubbery firm, measured $13 \times 10 \times 6 \mathrm{~cm}$, and was surrounded by a narrow rim of breast tissue. The cut surface showed focal areas of hemorrhage (Fig. 1). Microscopic examination showed a fibroepithelial tumor formed by an organoid pattern of regularly spaced ductal structures surrounded by dense and cellular fibrous stroma. There was no stromal overgrowth despite the cellularity of the stroma, with no evidence of necrosis. 


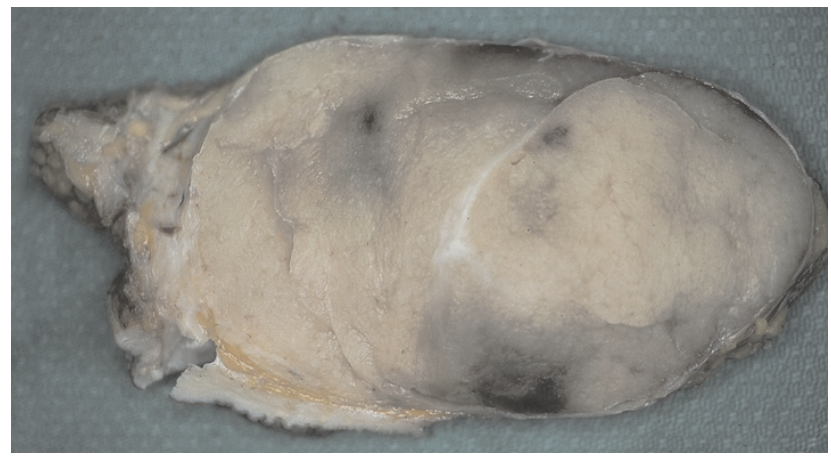

FIGURE 1. Gross appearance of primary tumor. Large, well-defined, lobulated mass showing areas of hemorrhage and a rim of normal breast tissue.

The margins were well circumscribed, with no evidence of an infiltrative border. The morphology of the stroma was quite striking. There were numerous arborizing, well-formed vascular channels that were present throughout the stroma (Fig. 2A). In some areas, this was associated with marked edema and extravasation of red blood cells. Lining of these vascular channels consisted of plump endothelial cells with no evidence of significant atypia or increased mitotic activity. This lining endothelium showed strong positivity for CD31, CD34, and Factor VIII (inset, Fig. 2B). The spindle cell fibroblastic stroma showed mild nuclear atypia, and the mitotic activity ranged from 1 to 2 per $10 \mathrm{HPF}$. These stromal cells showed strong positivity for vimentin and were negative for S-100. Staining with a low molecular weight keratin cam 5.2 was positive only in the ductal elements and completely negative in the stroma, ruling out the possibility of an unusual metaplastic carcinoma. The surrounding breast tissue showed lobular atrophy and stomal fibrosis. No significant epithelial changes were seen. Sections from the nipple were unremarkable, and the lesion

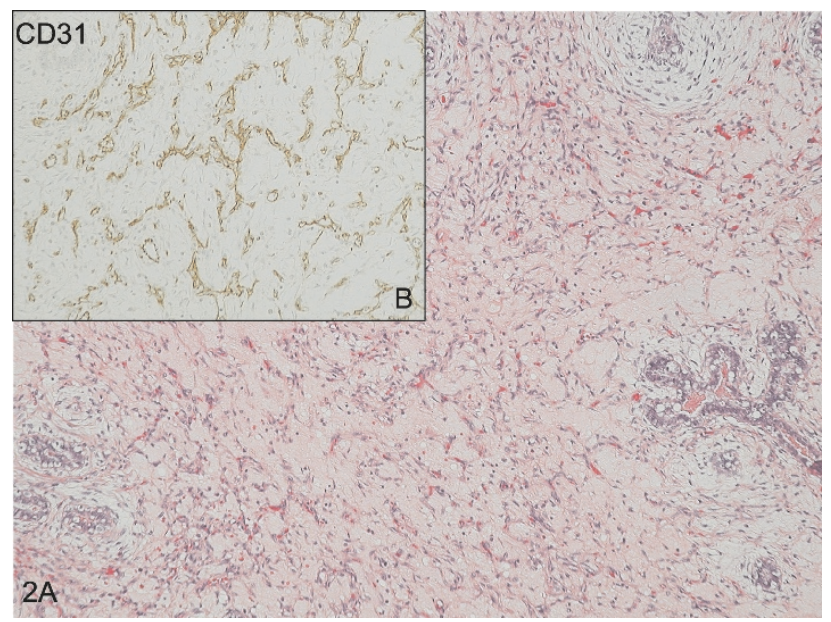

FIGURE 2. A-B, H\&E of primary tumor showing stromal cellularity with prominent benign vascular proliferation (CD31 stain in inset). Note the regular spacing of the epithelial component (magnification, 10×). appeared completely excised in the plane of the sections examined.

The recurrent tumor was large, soft, well-defined, and measured $10 \times 6 \times 4 \mathrm{~cm}$. The cut surface of the tumor was bulging, with a nodular contour, and it extended down to the fascia. Microscopic examination showed a variable appearance. In most areas, it had the characteristic appearance of malignant phyllodes tumor that had an extremely cellular stroma, with definite stromal overgrowth. The stroma was formed by highly atypical spindleshaped cells, with a mitotic rate that ranged from 5 to 6 per $10 \mathrm{HPF}$. There was a focal area showing a prominent network of vascular proliferation as seen in the primary tumor; however, the vascularity was not as diffuse as in the primary tumor (Fig. 3A-D). A malignant vascular component was not seen (Fig. $4 \mathrm{~A}-\mathrm{B})$. The other very unusual feature in this recurrent tumor was the marked atypical proliferation of the epithelial element, which had histological features for in situ carcinoma on H\&E sections (Fig. 5). The monotonous appearance of the cells favored lobular carcinoma in situ; however, E-cadherin expression in all the cells was more in keeping with that of ductal carcinoma in situ (inset, Fig. 5).

\section{Transmission Electron Microscopy}

Ultrastructural examination of the primary tumor confirmed the endothelial nature of the lining of the vascular channels in the stroma with the presence of intercellular junctions, basal lamina, pinocytotic vesicles and Weibel-Palade bodies.

\section{DISCUSSION}

The stromal cells in fibroadenomas are usually fibroblasts (1). Rare forms of stromal differentiation

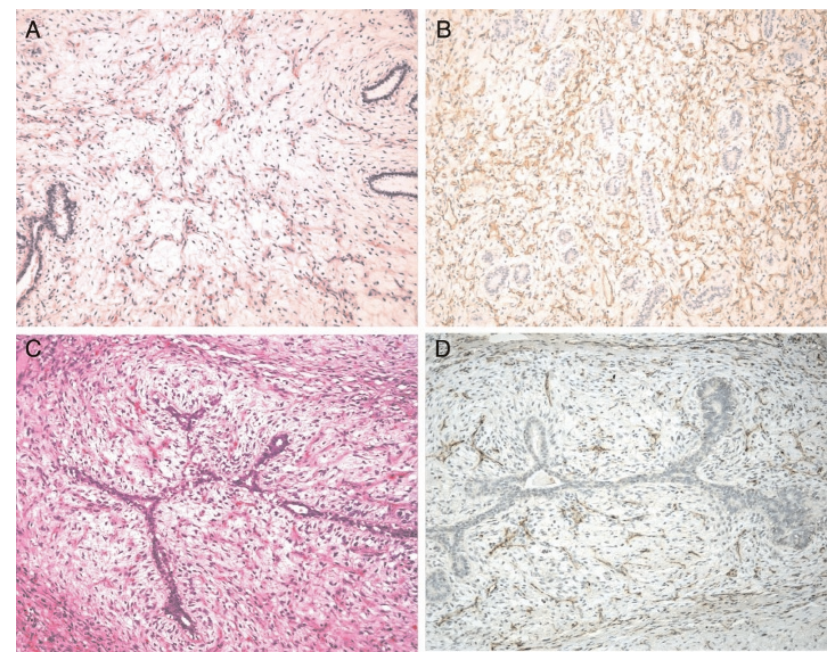

FIGURE 3. H\&E and CD31 staining of the primary illustrating the marked vascular proliferation (A, B) as compared with the reduced vascularity in the recurrence (C, D; magnification, $10 \times)$ 

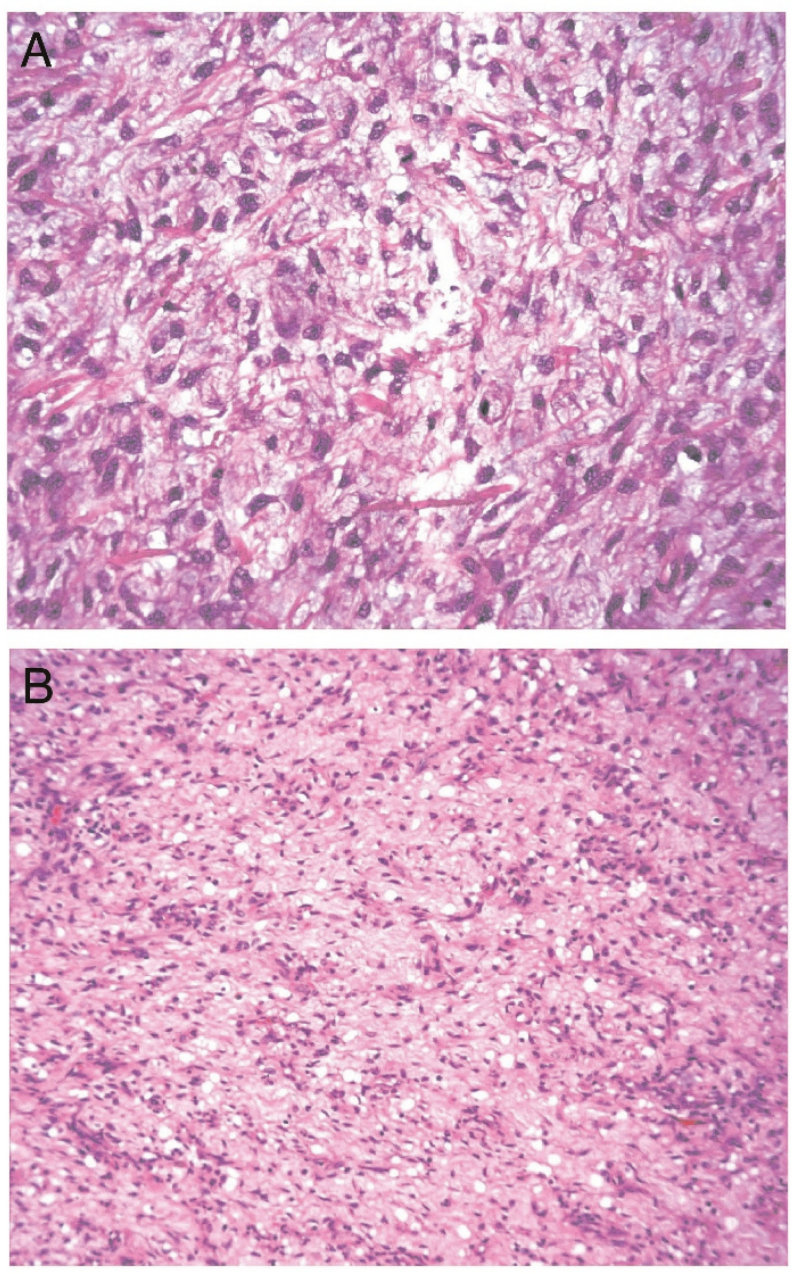

FIGURE 4. The predominantly spindle and atypical stroma of the recurrence (A) with (B) areas of prominent vascularity (magnification, $20 \times)$.

include smooth muscle metaplasia (2) or adipose differentiation (3).

Multinucleate stromal giant cells have also been noted in fibroadenomas and phyllodes tumors (4). Osteochondroid metaplasia of the stroma may also be encountered infrequently (5).

In our case, the primary tumor showed a fibroepithelial lesion with an unusual and peculiar stroma composed of extensive vascular proliferation throughout the tumor. The endothelial nature of the lining of these vascular channels was confirmed on immunohistochemistry and electron microscopic examination, which ruled out the possibility of extensive pseudoangiomatous hyperplasia. The slitlike pseudovascular spaces in pseudoangiomatous hyperplasia showed positivity for vimentin and CD34 but were negative for Factor VIII, CD31, or other endothelial markers (5-9). Ultrastructural examination of the lining of pseudoangiomatous hyperplasia showed an incomplete layer of fibroblastic cells. The pattern of vascular proliferation seen in the primary tumor did not resemble that of

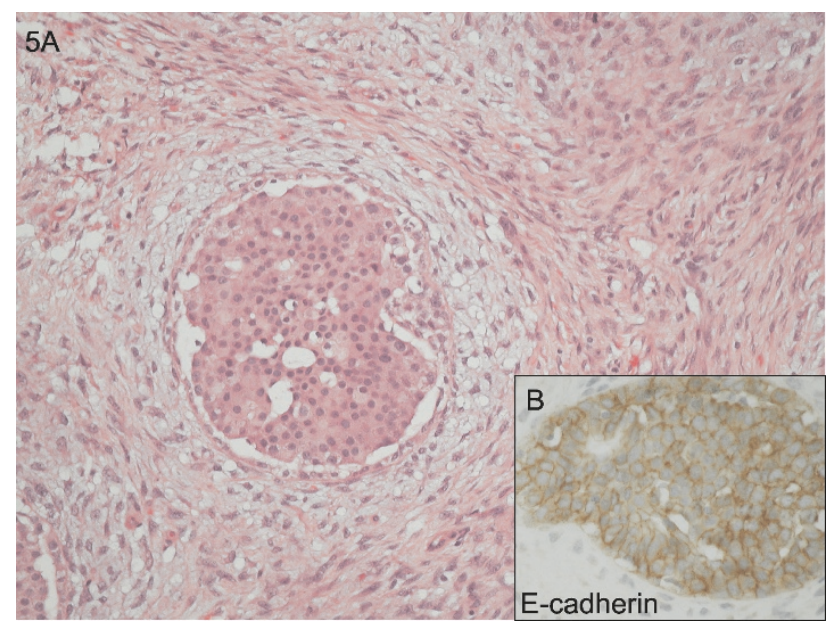

FIGURE 5. Recurrent tumor shows cellular stroma with atypical cells and mitosis surrounding a duct with in situ carcinoma (magnification, $40 \times$ ). Inset shows E-cadherin expression in the cells consistent with ductal carcinoma in situ (magnification, $40 \times$ ).

any previously described vascular lesion in the breast (10-18). A case of angiosarcoma but not benign vascular proliferation was reported in a case of phyllodes tumor (19). The primary tumor, though large in size, had only focal areas of cellular fibroblastic stroma, and stromal overgrowth was not observed as one would expect to see in a phyllodes tumor. The stroma was unusually vascular, with a benign arborizing network of capillaries present in many parts of the stroma. The primary fibroepithelial lesion could not be categorically classified as either phyllodes tumor or a fibroadenoma with cellular stroma because of this unusual feature. The recurrence, on the other hand, showed classic features of malignant phyllodes tumor that included stromal overgrowth, nuclear atypia, and a high mitotic rate. The role of vascular stroma in the pathogenesis and progression of both the epithelial and mesenchymal components is speculative. Recently, epithelial and mesenchymal interactions and in particular the role of angiogenesis in breast cancer has attracted the attention of investigators. Vascular stroma reportedly plays a role in the growth of the primary tumor as well as in invasion and metastasis in breast cancer (20). Angiogenesis and microvessel density have been implicated as important prognostic factors in breast cancer (21, 22). Da Silva et al. (23) found that the number of vessels counted in invasive ductal carcinoma was significantly higher than the number of vessels counted in fibroadenoma and in the adjacent breast tissue. However, others found that vascularity in breast carcinoma was similar to that of normal breast intralobular stroma (24). Valtola et al. (25) demonstrated that the expression of VEGFR-3 becomes up-regulated in the endothelium of angiogenic blood vessels in breast cancer. Wernert et al. 
(26) reported that genetic alterations (loss of heterozygosity and p53 mutation) occur in fibroblastic stroma of breast carcinoma, similar to those detected in the epithelial component, which indicates a possible role of tumor stroma in mammary carcinogenesis.

Recently, Tse et al. (27) found that there was a correlation between intratumoral vessel density and the histological grade when judged on CD-31stained slides and that microvessel density could be used as an additional histological parameter to distinguish between benign and malignant phyllodes tumor. Hasebe et al. (28) examined vascular endothelial growth factors (VEGF) in conventional fibroadenoma, cellular fibroadenoma, and phyllodes tumor and found that stromal cells in conventional fibroadenoma had the lowest frequency of VEGF protein expression and that those in phyllodes tumor had the highest frequency of expression. The belief that phyllodes tumor is a stromal neoplasm has recently been challenged. Both the stroma and the epithelium in phyllodes tumor can show distinct molecular changes, suggesting that both are part of the neoplastic process (29). It has also been observed that there is an interdependence of growth between the stromal and the epithelial components that explains the complex morphology seen in these tumors $(30,31)$. Stromal mitotic activity occurs in close proximity to the epithelial component, strengthening the role of stromal-epithelial interactions in progression of phyllodes tumor (30). The stromal-epithelial interaction can both be direct or systemic as a result of the release of angiogenic factor, particularly VEGF and VEGF receptor. This systemic effect might have played a role in our case as there was no preferential increased vascularity around the malignant epithelial component of the recurrent tumor.

Although the epithelial component in the primary tumor in our case did not show any significant atypia, the recurrence showed in situ ductal carcinoma. The vascular stroma might have played a role in the quick recurrence and malignant transformation of the epithelial and mesenchymal components. In situ carcinoma has been reported before in association with phyllodes tumor, and most have been in situ lobular carcinomas (32-34). Positive E-cadherin expression in our case, which had histologically equivocal features, confirmed the presence of ductal carcinoma in situ $(35,36)$.

This case appears to be a unique "aggressive giant" fibroepithelial lesion with cellular stroma showing abnormal vascular proliferation that recurred as malignant phyllodes tumor with in situ ductal carcinoma. The exuberantly vascular stroma in the primary tumor may have led to its quick progression to malignancy.

\section{REFERENCES}

1. Reddick RL, Shin TK, Sawhney D, et al. Stromal proliferations of the breast: an ultrastructural and immunohistochemical evaluation of cystosarcoma phyllodes, juvenile fibroadenoma, and fibroadenoma. Hum Pathol 1987;18:45-9.

2. Goodman ZD, Taxy JB. Fibroadenomas of the breast with prominent smooth muscle. Am J Surg Pathol 1981;5:99-101.

3. Powell CM, Rosen PP. Adipose differentiation in cystosarcoma phyllodes. A study of 14 cases. Am J Surg Pathol 1994;18:720-7.

4. Berean K, Tron VA, Churg A, et al. Mammary fibroadenoma with multinucleated stromal giant cells. Am J Surg Pathol 1986;10:823-7.

5. Spagnolo DV, Shilkin KB. Breast neoplasms containing bone and cartilage. Virchows Arch A Pathol Anat Histopathol 1983; 400:287-95.

6. Ibrahim RE, Sciotto CG, Weidner N. Pseudoangiomatous hyperplasia of mammary stroma. Some observations regarding its clinicopathologic spectrum. Cancer 1989;63:1154-60.

7. Vuitch MF, Rosen PP, Erlandson RA. Pseudoangiomatous hyperplasia of mammary stroma. Hum Pathol 1986;17:18591.

8. Powell CM, Cranor ML, Rosen PP. Pseudoangiomatous stromal hyperplasia (PASH). A mammary stromal tumor with myofibroblastic differentiation. Am J Surg Pathol 1995;19: 270-7.

9. Zanella M, Falconieri G, Lamovec J, et al. Pseudoangiomatous hyperplasia of the mammary stroma: true entity or phenotype? Pathol Res Pract 1998;194:535-40.

10. Hovorkova E, Ryska A, Spacek J. Pseudoangiomatous hyperplasia of mammary stroma. Cesk Patol 1998;34:109-15.

11. Jozefczyk MA, Rosen PP. Vascular tumors of the breast. II. Perilobular hemangiomas and hemangiomas. Am J Surg Pathol 1985;9:491-503.

12. Lesueur GC, Brown RW, Bhathal PS. Incidence of perilobular hemangioma in the female breast. Arch Pathol Lab Med 1983;107:308-10.

13. Rosen PP. Vascular tumors of the breast. V. Nonparenchymal hemangiomas of mammary subcutaneous tissues. Am J Surg Pathol 1985;9:723-9.

14. Rosen PP. Vascular tumors of the breast. III. Angiomatosis. Am J Surg Pathol 1985;9:628-52.

15. Rosen PP, Jozefczyk MA, Boram LH. Vascular tumors of the breast. IV. The venous hemangioma. Am J Surg Pathol 1985; 9:659-65.

16. Calcada CP, Cabanelas B, Figueiredo P, et al. Angiosarcoma of the breast. Acta Med Port 1994;7:555-9.

17. Christodoulakis M, Gontikakis E, Giannikaki E, et al. Primary angiosarcoma of the breast. Eur J Surg Oncol 1998;24:76-8.

18. Hoda SA, Cranor ML, Rosen PP. Hemangiomas of the breast with atypical histological features. Further analysis of histological subtypes confirming their benign character. Am J Surg Pathol 1992;16:553-60.

19. Rosen's breast pathology. 2nd ed. Philadelphia, PA: Lippincott, Williams and Wilkins; 2001. p. 184 and 187.

20. Brown LF, Guidi AJ, Schnitt SJ, et al. Vascular stroma formation in carcinoma in situ, invasive carcinoma, and metastatic carcinoma of the breast. Clin Cancer Res 1999;5:1041-56.

21. Chu JS, Huang CS, Chang KJ. The prognostic significance of tumor angiogenesis in Taiwanese patients with invasive ductal breast carcinomas. Cancer Lett 1998;134:7-14.

22. Hansen S, Grabau DA, Sorenson FB, et al. Vascular grading of angiogenesis and prognostic significance in breast cancer. Br J Cancer 2000;82:339-47.

23. Da Silva ID, De Lima GR, Gebrim LH, et al. Invasive ductal carcinoma, fibroadenoma and adjacent breast stroma angiogenesis: an immunohistochemical and morphometrical study. Bull Assoc Anat (Nancy) 1996;80:17-9. 
24. Schor AM, Pendleton N, Pazouki S, et al. Assessment of vascularity in histological sections: effects of methodology and value as an index of angiogenesis in breast tumours. Histochem J 1998;30:849-56.

25. Valtola R, Salven P, Heikkila P, et al. VEGFR-3 and its ligand VEGF-C are associated with angiogenesis in breast cancer. Am J Pathol 1999;154:1381-90.

26. Wernert N, Locherbach C, Wellman A, et al. Presence of genetic alterations in microdissected stroma of human colon and breast cancers. Anticancer Res 2001;21:2259-64.

27. Tse GM, Ma TK, Chan KF, et al. Increased microvessel density in malignant and borderline mammary phyllodes tumor. Histopathology 2001;38:567-70.

28. Hasebe T, Imoto S, Sasaki S, et al. Proliferative activity and tumor angiogenesis is closely correlated to stromal cellularity of fibroadenoma: proposal fibroadenoma, cellular variant. Pathol Int 1999;49:435-43.

29. Sawyer EJ, Hanby AM, Ellis P, et al. Molecular analysis of phyllodes tumors reveals distinct changes in the epithelial and stromal components. Am J Pathol 2000;156:1093-8.
30. Sawhney N, Garrahan N, Douglas-Jones AG, et al. Epithelial-stromal interactions in tumors. A morphologic study of fibroepithelial tumors of the breast. Cancer 1992; 70:2115-20.

31. Sawyer EJ, Hanby AM, Rowan AJ, et al. The Wnt pathway, epithelial-stromal interactions, and malignant progression in phyllodes tumors. J Pathol 2002;196:437-44.

32. Grove A, Deibjerg Kristensen L. Intraductal carcinoma within a phyllodes tumor of the breast: a case report. Tumori 1986;72:187-90.

33. Padmanabhan V, Dahlstrom JE, Chong GC, et al. Phyllodes tumor with lobular carcinoma in situ and liposarcomatous stroma. Pathology 1997;29:224-6.

34. Knudsen PJ, Ostergaard J. Cystosarcoma phyllodes with lobular and ductal carcinoma in situ. Arch Pathol Lab Med 1987;111:873-5.

35. Acs G, Lawton TJ, Rebbeck TR, et al. Differential expression of E-cadherin in lobular and ductal neoplasms of the breast and its biological and diagnostic implications. Am J Clin Pathol 2001;115:85-98. 